\title{
MAINTAINING MICRON-SIZE BEAMS IN COLLISION AT THE INTERACTION POINT OF THE STANFORD LINEAR COLLIDER-
}

\author{
F. Rouse, ${ }^{(a, b)}$, T. Gromme, W. Kozanecki, and N. Phinney \\ Stanford Linear Accelerator Center, Stanford University, Stanford, CA 94309 USA
}

\begin{abstract}
In order to maintain collisions between two micronsize beams at the interaction point of the SLC, we take advantage of the mutual electromagnetic deflection induced by one beam on the other as they cross with a nonzero relative impact parameter. We determine simultaneously the incoming and outgoing trajectory parameters of each beam on a pulse-by-pulse basis, using beam position monilors located near the IP. Comparing incoming and outgoing angles for a given beam yields the magnitude of the deflection the beam experienced during the collision from which the distance currently separating the two beams can be extracted. A simple proportional control is applied to calculate the change in upstream corrector settings to null] out this distance.
\end{abstract}

\section{INTRODUCTION}

The SLAC Linear Collider (SLC) is a novel accelerator produces $e^{+} e^{-}$collisions at center-of-mass energies around the mass of the neutral intermediate vector boson $Z^{0}$. The collisions occur between electrons and positrons produced on every crossing, as opposed to being stored for an extended time as in electron-positron storage rings. However, since the accelerator produces fewer particles per bunch and the collision frequency is much less than at storage rings, the beams that collide in the SLC must have exiremely small spot sizes in order to produce a usable number of interactions. Controlling and measuring the beam sizes and positions at the micron level is essential. We describe in this paper the performance of a leedback loop that stabilizes the transverse positions of the beams at the interaction point (IP).

\section{BEAM-BEAM DEFLECTIONS}

When electrons and positrons are brought inco collisions. they mutually deflect each other due to the electromagnetic interaction between them $[1,2]$. We measure the deflection with Beam Position Monitors (BPMs) located upstream and downstream of the IP for both beams. The incoming and outgoing beam trajectories are fit for the electrons and positrons separately [3] with the constraint that for each beam, the incoming and outgoing orbit share a common transverse position at the IP.

Figure 1 shows an example of an observed beambeam deflection in the $x$ plane. The data was taken during a beam-beam scan. We scan the electron beam across the positron beam in two micron steps. Note that the positron deflection is opposite to that for electrons, as expected. The deflections are not equal, because the intensity of the electron beam is close to twice that of the positron beam.

* Work supporied in part by Department of Energy contracts DE-AC03-76SF00515 (SLAC) and DE-AC03-76SF00098 (SLAC)

(a) Iniversity of Cajifornia, Lawrence Berkeley Laboratory, Berkeley, CA 94720

(b) Present address: University of California al Davis.

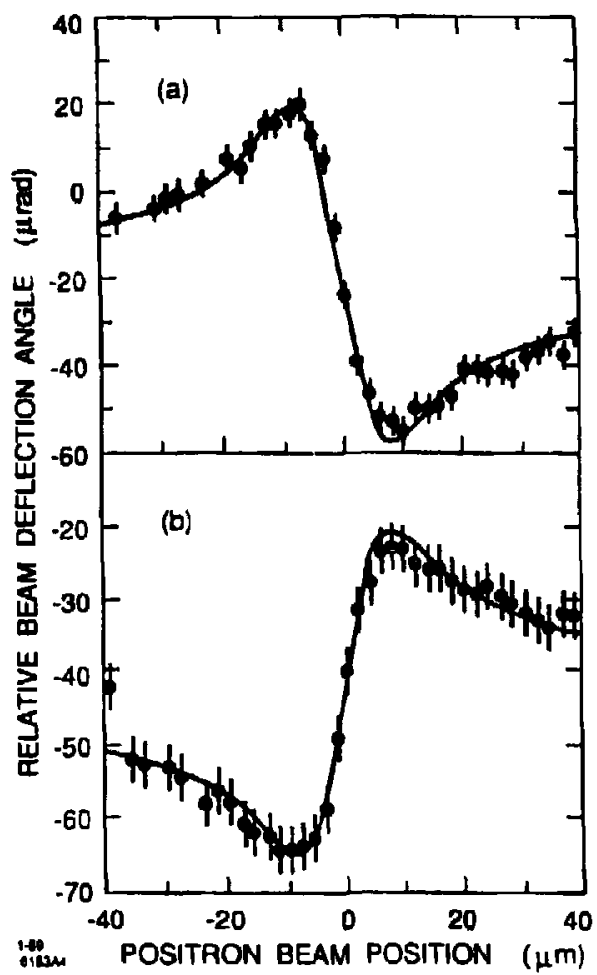

Figure I. Deflection experienced by positrons (top) and electrons (bottom) plotted versus the transverse distance between the two beams. The curve is fit to the data in the round beam approximation. The background deflection is the value of the deflection angle at zero distance between the two beams, and is determined by a fit to the dala.

\section{PULSE-TO-PULSE MONITORING}

We define the $z$ direction as along the direction of motion of the electrons, $x$ the horizontal axis, and $y$ the vertical axis. Throughout this paper, we refer to the 2 plane; the same comments and equations apply equally to the $y$ plane.

We parameterize the trajectory through the IP as a function of the $x$ position at the IP, and of the incoming and defiection angles in the $\boldsymbol{z}$ plane. We then determisu. the least square fit to the trajectory [3]. This procedure is carried out online by an Intel 80386 microprocessor.

Figure 2 shows the deflection angles derived from the fitted electron trajectory. The expected resolution on the deflection angle, neglecting beam motion, is $2.5 \mu \mathrm{rat}$ for a BPM position resolution of $10 \mu \mathrm{m}$. The resolution determined by fitting a Gaussian to llie projectiou 


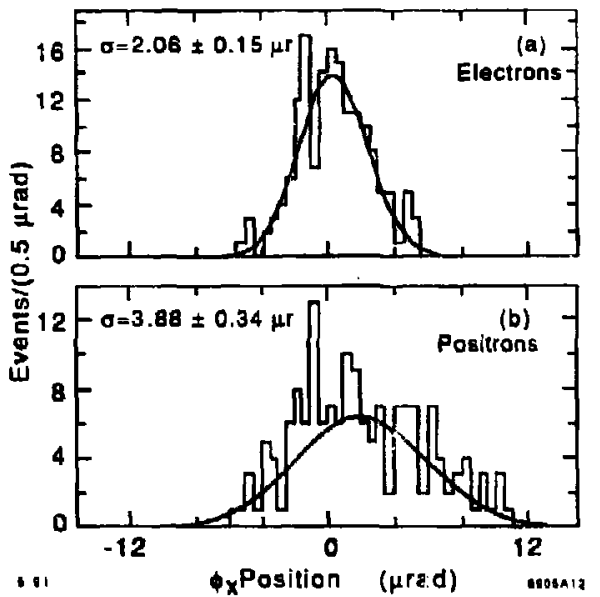

Figure 2. The distribution of deflection angles derived from then fitted electron (a) and pasitron (b) beam trajectories. The plot is a projection onto the $y$-axis of the deflection angle versus time plot.

onto the $y$ axis of reveals (a) a slightly better and (b) a slightly worse resolution. Neither result is inconsistent with expectations.

We have verified that the difference in deflections experienced by the electron and positron beams can be explained by the difference in intensity of the two beams. The deflection angle of one beam is directly proportional to the intensity of the other [1]. Therefore, we consider the intensity-normalized deflection angle difference between the electron and positron beam:

Difference $=\left[\left(N_{\mathrm{a}} \phi_{\mathrm{a}}-\right) / N_{\mathrm{a}}+\right]-\left[\left(N_{\mathrm{a}} \phi_{\mathrm{a}}+\right) / N_{\mathrm{e}-}\right]$ where $N_{e}$ - is the number of electrons, $N_{e}+$ is the number of positions, and $N_{a}$ is the average of the two. When we plot the distribution of differences and fit it to a Gaussian, we fusd the centroid of the distribution to be at zero, within errors, as shown in Fig. 3.

\section{FEEDBACK}

The deflection curve shown in Fig. 1 becomes approximately linear when the two beams are very close to one another. The slope at crossover in the horizontal plane, obtained as the first term in the Taylor expansion in the limit of small $\boldsymbol{\Sigma}_{x}$ is

$$
S_{x}=\left[\left(-2 r_{e} N_{t}\right) / \gamma\right] \times 1 /\left[\Sigma_{x}\left(\Sigma_{z}+\Sigma_{y}\right)\right] \text {. }
$$

This slope in a given plane ( $x$ or $y$ respectively) depends strongly on the "in-plane" beam size $\Sigma_{x}\left(\Sigma_{y}\right)$, and somewhat more weakly on the "out-of-plane" size $\Sigma_{y}\left(\Sigma_{x}\right)$. The quantity $N_{1}$ is the intensity of the target beam. We define the quantity

$$
S_{x}^{\prime}=S_{s} / N_{t}
$$

as the intensity-normalized slope of the deflection curve.

The beam-beam deflection is quite accurately described by a single slope parameter very elose to the crossover point. This approximation breaks down as the distance between the two beams increases. However, our

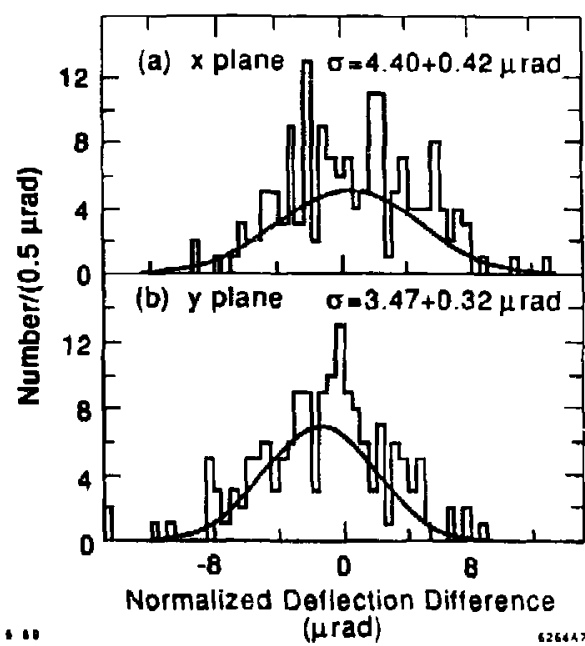

Figure 3. The difierence between intensity-normalized de. flections in (a) the $I$ and (b) the $y$ planes.

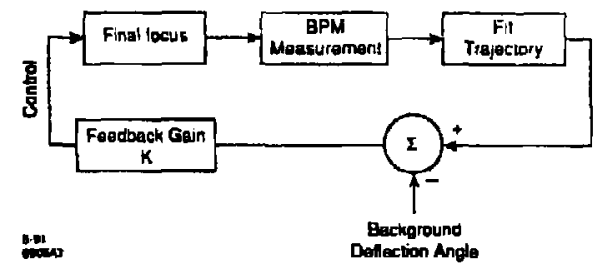

Figure 4. Conceptualization of the proportional feedback system used to maintain the beams in collisions.

theoretical results have indicated that for impact parameters on the order of one beam size, the difference beiveen the linear approximalion and the actual deflection is only. of the order of $10 \%$.

We measure the intensity-normalized slope for the beam deflections periodically by performing a full-bean scan [2]. The BPMs measure the intensity of both beanis pulse-by-pulse. We can therefore correct for changes in the intensity of the lwo beans over time. We compute the distance between the two beams $\Delta_{x}$ by dividing the currentlydetermined deffection angle by the intensity-normalized slope and the measured number of particles in the target beam,

$$
\Delta_{r}=\phi_{x} /\left(N_{t} S_{x}^{\prime}\right) \text {. }
$$

\section{RESULTS}

The simple proportional feedback system illustrated in Fig. 4 shows how we maintain the beams in collision. We compute the required change in magnet getlings of upstream air-core correctors in order to null out any movisment between the beams. The corrector magnets are extremely fast and can come to their new settings within $1 / 120$ of a second. The magnets only have a $100 \mu \mathrm{m}$ raugc. but as we can see from Fig. 1, this is sufficient to returu the beams to collision over the range of deflection distancus we observe. 


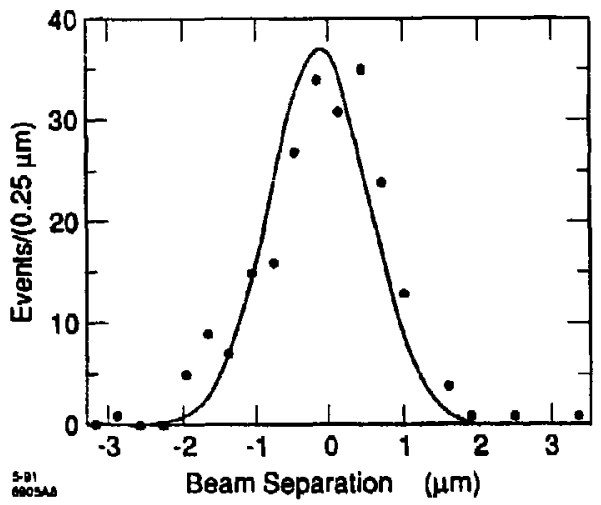

Figure 5. The distribution of beam-beam separations over a period of twenty-four seconds.

The gain of the closed-loop feedback is the only parameter that we use to control the response of the feedback loop. We plot the distribution of distances between the beams over a twenty-four second period with a feedback gain of 0.3 in Fig. 5. If we fit this distribution to a Gaussian, plus a constant background, we find the centroid of the Gaussian is $-0.16 \pm 0.06 \mu \mathrm{m}$ and the width of the Gaussian is $0.68 \pm 0.09 \mu \mathrm{m}$. The background was found to be zero, within errors. We repeated this experiment for several gains. In Fig. 6 we plot the average of the $\sigma$ s as a function of gain. It is obvious that at a gain of 0.4 , the feedback lcop has significantly worse performance than for gains between 0.2 and 0.3 . We therefore conclude the oprimal gain of the system is of the order of 0.3 .

\section{CONCLUSION}

This paper has presented the performance of a feedback loop intended to maintain the electron and posit ron beams in collision at the Stanford Linear Collider. The loop was commissioned in early 1990, and was part of the usual operation by the fall. The loop brings the beams back into collision in two pulses for separations of $6 \mu \mathrm{m}$, with a closed loop gain of about 0.3 . The width of distribution of beam-beam separation distances is about $0.6 \mu \mathrm{m}$.

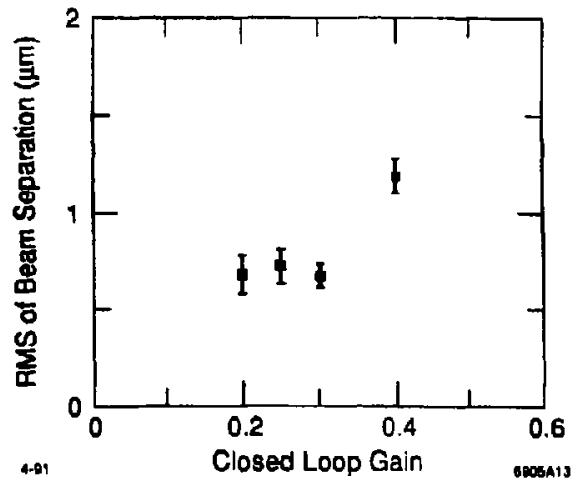

Figure 6. The average RMS of the beam-beam separations, as a function of gain.

and the centroid of distances averages $-0.15 \mu \mathrm{m}$. We estimate that this feedback loop increases the luminosity of the SLC by a factor of 20 to $30 \%$ over what it would be in the absence of the loop.

\section{ACKNOWLEDGMENTS}

The authors thank P. Bambade and R. Erikson for original ideas on the use of beam-beam deflections as a diagnostic tool. We also thank W. Koska and S.R. Wagner for their extensive theoretical and experimental work in applying those ideas to the SLC. One of the authors (F.R.) would like to thank the University of California Presidential Fellowship program for support during this work. Thanks also to L. Hendrickson for her software efforts.

\section{REFERENCES}

[1] P. Bambadc et al., "Beam-Beam Deflections as an Interaction Point Diagnostic for the SLC." Phys. Rev. Lett. 62. no. 25 (1989); SLAC-PUB-3979.

[2] W. Koska et al., "Beam-Beam Deflection as a Beam Tuning Tool at the SLAC Linear Collider," in Proc. 1989 IEEE Particle Accelerator Conf., Chicago IL; also Nucl. Instrum. Methods A286, 32 (1990).

[3] W. Koska and S. Wagner, "Beam Trajectory Determination at the IP," SLAC-CN-365 (1988). 